\title{
Altered polar character of nanoconfined liquid water
}

\author{
Sayantan Mondal 1 , Subhajit Acharya, and Biman Bagchi ${ }^{*}{ }^{*}$ \\ Solid State and Structural Chemistry Unit, Indian Institute of Science, Bengaluru, India
}

(Received 26 February 2019; revised manuscript received 3 June 2019; published 3 December 2019)

\begin{abstract}
Atomistic molecular dynamics simulation study of dipolar fluids confined to spherical nanocavities of radii ranging from $R_{c}=1$ to $4 \mathrm{~nm}$ reveals a surprisingly small Kirkwood correlation factor $\left(g_{K}\right)$ in water, but not so in dipolar Stockmayer fluid. This results in an ultrafast relaxation of the total dipole moment time correlation function (DMTCF) of water. The static dielectric constant of water under nanoconfinement exhibits a strong dependence on size with a remarkably low value even at $R_{c}=3 \mathrm{~nm}$ and a slow convergence to the bulk value because of surface-induced long-range orientational correlations. Interestingly, DMTCF exhibits a bimodal $1 / f$ noise power spectrum.
\end{abstract}

DOI: 10.1103/PhysRevResearch.1.033145

\section{INTRODUCTION}

A large number of physicochemical processes occur in aqueous solution under nanoconfinement. Confinement can significantly modulate the phase behavior, ion transport, reaction pathways, and chemical equilibrium [1-3]. For example, confined water exhibits enhanced self-dissociation [4]. This increases the ionic product and affects other physicochemical properties. Electrospray experiments reveal a marked enhancement in the reaction rate in aqueous droplet medium $[5,6]$. Some reactions adopt different mechanisms that lead to unexpected products [7]. Experiments often find slower relaxation in confined water, although the reverse has also been observed [8-12]. Also, the altered polar character of confined water controls many other important processes such as solvation and charge transfer [13]. Phenomenological theories invoke a continuum model with a given dielectric constant of the liquid $[13,14]$. Hence, understanding the dielectric behavior of confined dipolar liquids becomes important to comprehend these processes [15-20]. Although the dielectric properties of bulk dipolar liquids are well understood [21,22], only a limited number of theoretical studies have been devoted to understand the same under spherical nanoconfinement [18,23,24].

The Hamiltonian and total interaction potential for confined liquid systems is given by Eq. (1).

$$
\begin{aligned}
H & =K_{\text {liquid }}^{(0)}+U(\{\boldsymbol{r}\}, \boldsymbol{R}) ; \\
U(\{\boldsymbol{r}\}, \boldsymbol{R}) & =\sum_{i, j(>i)} u_{i j}\left(\boldsymbol{r}_{i j}\right)+\sum_{i, k} u_{i k}\left(\boldsymbol{R}_{i k}\right) .
\end{aligned}
$$

Here, $K_{\text {liquid }}^{(0)}$ denotes kinetic energy, $u_{i j}\left(\boldsymbol{r}_{i j}\right)$ represents intermolecular interactions, and $u_{i k}\left(\boldsymbol{R}_{i k}\right)$ represents the liquid-

\footnotetext{
*Corresponding author: bbagchi@iisc.ac.in

Published by the American Physical Society under the terms of the Creative Commons Attribution 4.0 International license. Further distribution of this work must maintain attribution to the author $(s)$ and the published article's title, journal citation, and DOI.
}

surface interaction. In practice, one models $u_{i j}\left(\boldsymbol{r}_{i j}\right)$ as the sum of electrostatic, dipolar, and Lennard-Jones interactions. However, $u_{i k}\left(\boldsymbol{R}_{i k}\right)$ is modeled in different ways to characterize different surfaces.

The combined effects of geometric confinement and surface-liquid interactions have remained a subject of discussions for many decades [25-27]. In water, both the effects are complex. Liquid water strives to maintain its hydrogen bond network (HBN) to minimize free energy. This is termed as the principle of minimal frustration $[28,29]$. As water exhibits several anomalies, we study another model dipolar liquid to establish general perspectives.

We ask the following questions: (i) How does the static dielectric constant $(\varepsilon)$ scale with the size of the nanocavity? (ii) What is/are the microscopic origin(s) of ultrafast collective orientational relaxation under nanoconfinement? (iii) How does the surface-liquid interaction affect the structure and dynamics of confined liquid? We aim to answer these questions in the present work.

\section{THEORETICAL FORMALISM}

For spherical samples, the generalized Clausius-Mossotti relation provides the only exact expression for frequencydependent dielectric function, $\varepsilon(\omega)$ [Eq. (2)].

$$
\frac{\varepsilon(\omega)-1}{\varepsilon(\omega)+2}=\frac{4 \pi}{3 V} \alpha(\omega)
$$

Here, $V$ denotes the volume and $\alpha(\omega)$ represents the frequency-dependent macroscopic polarizability [30,31]. By using the linear response theory (LRT) of Kubo [32], $\alpha(\omega)$ can be expressed in terms of the after-effect function, $b(t)$, which is related to the dipole moment time correlation function (DMTCF) [Eq. (3)]

$$
\alpha(\omega)=-\int_{0}^{\infty} d t e^{i \omega t} \frac{d b(t)}{d t} ; \quad b(t)=\frac{1}{3 k_{B} T}\langle\boldsymbol{M}(0) \boldsymbol{M}(t)\rangle .
$$


By using Eqs. (2) and (3) one can transform Eq. (4) in the $\omega=0$ limit [Eq. (4)].

$$
\frac{\varepsilon-1}{\varepsilon+2}=\frac{4 \pi}{9 V k_{B} T}\left\langle M^{2}\right\rangle_{S} .
$$

This is the well-known Clausius-Mossotti equation which is exact but has the constraint of having to be immersed in a medium of dielectric constant unity. Recently, an interesting approach was employed to obtain spatially resolved dielectric function in spherical geometry [33].

We note that, for a virtual sphere of radius $r_{0}$ inside a larger spherical domain of radius $R_{c}$, Berendsen had earlier derived an expression for $\left\langle M\left(r_{0}\right)^{2}\right\rangle$ [Eq. (5)], the mean-squared dipole moment of the virtual sphere $[18,34]$.

$$
\frac{\left\langle M\left(r_{0}\right)^{2}\right\rangle}{3 k_{B} T r_{0}{ }^{3}}=\frac{(\varepsilon-1)}{9 \varepsilon(\varepsilon+2)}\left[(\varepsilon+2)(2 \varepsilon+1)-2(\varepsilon-1)^{2}\left(\frac{r_{0}}{R_{c}}\right)^{3}\right] \text {. }
$$

However, Berendsen's approach assumes that the dielectric constant of the region $r \in\left[0, r_{0}\right]$ to be the same as the outer shell, $r \in\left(r_{0}, R_{c}\right]$. This condition may not hold in the nanoscopic world. Equation (5) reduces to Eq. (4) for $r_{0}=R_{c}$ and to the Onsager-Kirkwood relation [22] for $R_{c} \rightarrow \infty$. One can of course obtain $\left\langle M^{2}\right\rangle_{S}$ from simulations. However, the use of Eq. (5) in the nanoworld remains questionable. In the Supplemental Material (SM S1), we detail the derivation of Eq. (5) with a discussion on its applicability [35].

On the other hand, $\varepsilon$ for a rectangular box of liquid with periodic boundary condition (PBC), is given by Eq. (6) if LRT is applied [36,37].

$$
\varepsilon=1+\frac{4 \pi}{3 V k_{B} T}\left\langle M^{2}\right\rangle_{\mathrm{PBC}} .
$$

Use of PBC introduces approximations. $\varepsilon$ calculated from Eq. (6) approaches the bulk value even for small-sized systems as it contains the effect of PBC. While the ClausiusMossotti equation is exact, the calculation of $\varepsilon$ of the medium inside the sphere requires the creation of a restricting surface and requires the use of definitive surface-liquid interactions. While use of PBC partly avoids this difficulty, the effects introduced by $\mathrm{PBC}$ to render this approach are also approximate.

Another constraint, not restrictive for large systems but serious in the nanoscopic world, is the determination of the effective volume. As shown later, the Clausius-Mossotti relation shows a strong sensitivity to the volume. Here, volume is determined by the nature of the surface-liquid interactions. For soft-repulsive walls the interaction excludes a portion of the volume. This poses a problem of far-reaching consequences. Usually in statistical mechanics, we probe the volume $(V)$ from outside without considering the solute-solvent interactions. Of course, the goal of statistical mechanics is to consider the limit of $V \rightarrow \infty$ to recover the thermodynamic properties. However, that limit becomes inapplicable in the nanoworld [38]. In some earlier studies, the volume of the cavity was not estimated systematically $[18,39]$. Therefore, the values obtained remain doubtful. We address and resolve this issue in SM S2 [35].
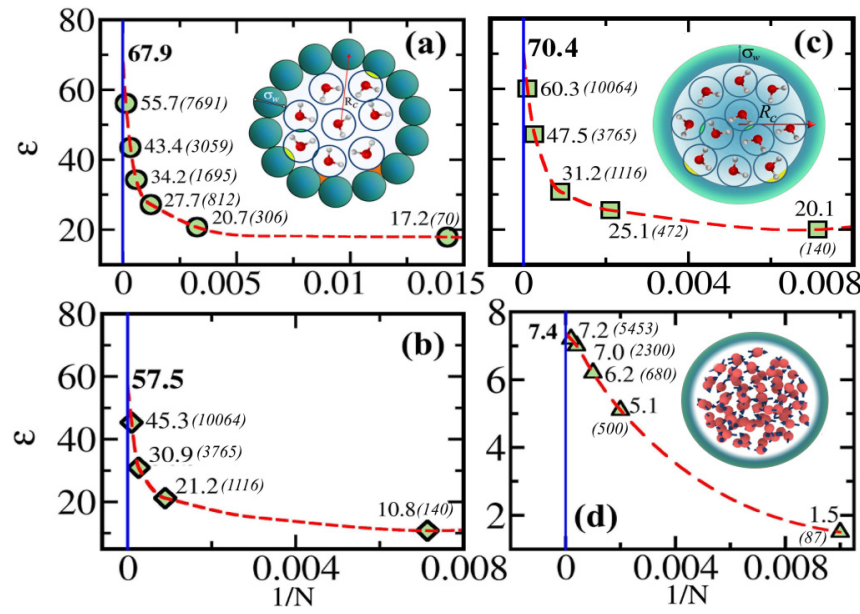

FIG. 1. Static dielectric constant against the inverse of the number of molecules $(1 / N)$ for aqueous nanocavities with (a) LJ-12,6 atomistic walls, (b) LJ-10,4,3 walls, (c) LJ-9,3 walls, and (d) SF with LJ-9,3 walls. The convergence for water is extremely slow. Extrapolations using a cubic polynomial provide asymptotic values of 67.9, 57.5, and 70.4, respectively (thermodynamic limit). However, the convergence for SF is remarkably fast. Insets: Schematic two-dimensional cross sections of the nanocavities. Penetration of water molecules into the soft spheres (yellow regions) and inaccessible regions (orange) inside the cavity invoke errors in the volume calculation. Data represented as $\left[\varepsilon\left(N_{\text {wat }}\right)\right]$ (SM S4 and S5 [35]).

We perform atomistic molecular dynamics simulations of spherical nanocavities filled with extended simple point charge model (SPC/E) water or Stockmayer fluid (SF), suspended in vacuum and enclosed by rigid nonpolarizable walls. For water we consider three different liquid-surface interactions: (i) LJ-12,6 (where LJ is Lennard-Jones) potential with atomistic wall, (ii) LJ-9,3 potential, and (iii) LJ-10,4,3 potential (see SM S3 [35] for simulation details).

\section{RESULTS AND DISCUSSION}

Dielectric response exhibits anisotropy under confinement $[20,40,41]$. In a spherical cavity one divides the field vector into longitudinal (azimuthal and polar) and transverse (radial) components. Here, we calculate the overall, average, and macroscopic dielectric response $\varepsilon$. Electrostatic screening, which controls the encounter probability among solutes, is controlled by $\varepsilon$ of the medium. We report $\varepsilon$ of water and $\mathrm{SF}$ in spherical nanoconfinements by employing Eq. (4) and use the effective/accessible volume. We report the calculated values in Fig. 1 where inside brackets the numbers of water molecules are mentioned.

We find that $\varepsilon$ of nanoconfined water shows a strong size dependence and slow convergence to the bulk value, unlike SF [Fig. 1(d)]. For water, even inside a 3 -nm cavity the value of $\varepsilon$ remains remarkably low $(\sim 40)$, compared to the bulk $(\sim 68$ for SPC/E water). To obtain $\varepsilon$ in the thermodynamic limit $(N \rightarrow \infty)$, we plot $\varepsilon$ against $1 / N$ (Fig. 1). Extrapolations provide quantitative agreements for the bulk, as shown in Fig. 1. 

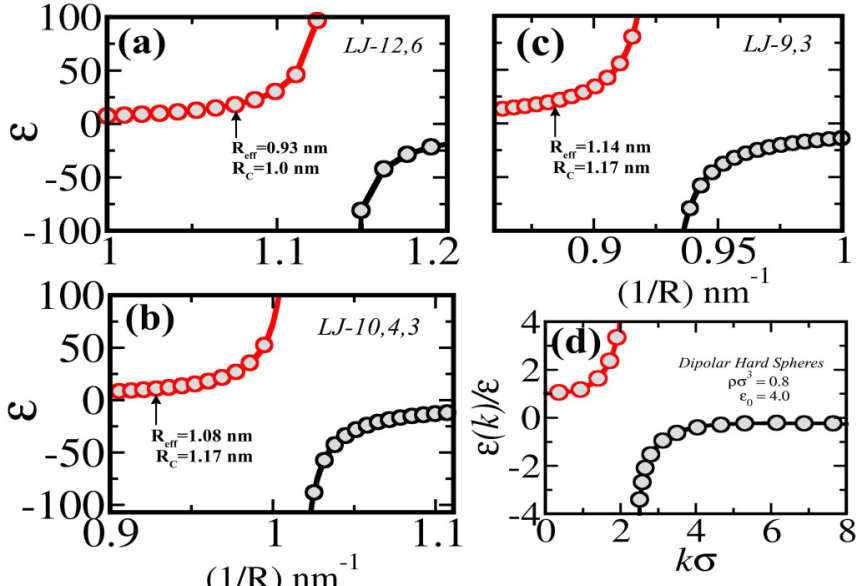

FIG. 2. $\varepsilon$ of water against the inverse of effective radius aqueous nanocavities. Surfaces are described by (a) LJ-12,6, (b) LJ-10,4,3, and (c) LJ-9,3 potentials. The sensitivity of $\varepsilon$ to $R_{\text {eff }}$ is strong. After the divergentlike behavior $\boldsymbol{\varepsilon}$ becomes negative. (d) Wavevector $(k)$-dependent dielectric function calculated for dipolar hardsphere liquid using mean spherical approximation shows similar divergencelike behavior (replotted from Ref. [42]).

In order to further analyze the size dependence, we rearrange Eq. (4) to obtain Eq. (7).

$$
\varepsilon=\frac{8 \pi \beta\left\langle M^{2}\right\rangle+9 V}{9 V-4 \pi \beta\left\langle M^{2}\right\rangle} .
$$

Clearly, $\varepsilon$ diverges if $9 V=4 \pi \beta\left\langle M^{2}\right\rangle$ (Fig. 2). In periodic/macroscopic systems, volume calculation is error-free. However, it becomes nontrivial in nanoconfined systems, especially when the liquid is surrounded by soft-repulsive walls.

We determine the volume using statistical mechanics. That is, we first obtain radial distributions of water molecules with respect to the center of the sphere (SM S2) [35]. We then integrate over the density distribution and normalize to the total number $\left(N_{\text {wat }}\right)$.

$$
\frac{1}{N_{\text {wat }}} \int_{0}^{R_{\text {eff }}} d r 4 \pi r^{2} \rho(r)=1 .
$$

We use Eq. (8) to obtain $R_{\text {eff }}\left(<R_{C}\right)$ numerically. This provides a measure of effective volume, $V_{\text {eff }}=(4 / 3) \pi R_{\text {eff }}^{3}$. We demonstrate the sensitivity of $\varepsilon$ to $V_{\text {eff }}$ in Figs. 2(a) $-2(\mathrm{c})$. For same $\left\langle\delta M^{2}\right\rangle$, we vary the radius from $R_{c}-\left(\sigma_{W} / 2\right)$ to $R_{c}$ for $1-\mathrm{nm}$ aqueous nanocavities.

Figure 2 shows that a small error in $R_{\text {eff }}$ can significantly alter $\varepsilon$. For example, if one changes $R_{\text {eff }}$ from 0.93 to $0.90 \mathrm{~nm}$, $\varepsilon$ changes from 17.2 to 46.1 . Hence, careful determination of $V_{\text {eff }}$ becomes crucial. However, we do not observe such a strong dependence on volume for confined SF. This is attributed to the absence of extensive HBN. By employing mean spherical approximation, Chandra and Bagchi obtained similar divergencelike behavior of wave-number-dependent dielectric function $[\varepsilon(k)]$ in a dipolar hard-sphere liquid [Fig. 2(d)] [42].

Rotational motions of solvent are important for solvation. The initial ultrafast decay $(\sim 60 \%-80 \%)$ in aqueous solvation dynamics is attributed to several factors: (i) librational modes
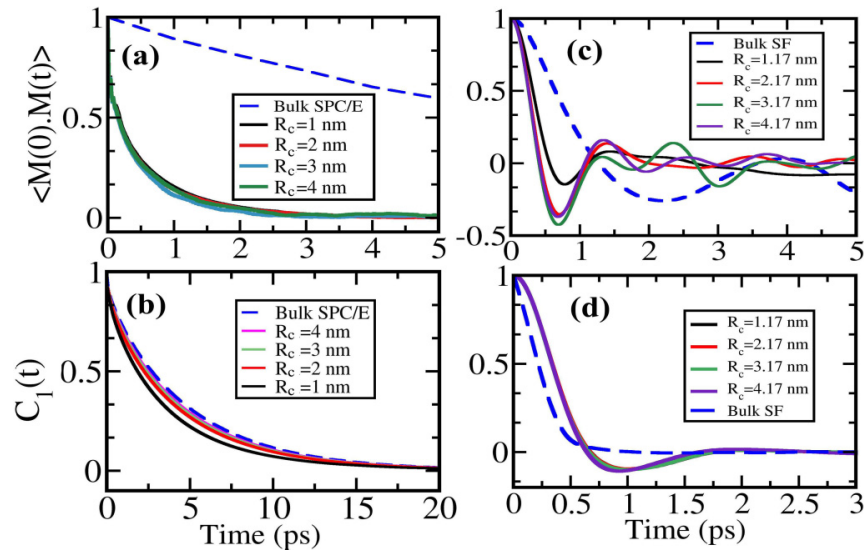

FIG. 3. (a) Total DMTCF of water inside spherical nanocavities. The relaxation under confinement is approximately 20 times faster than the bulk (dashed line). (b) First rank orientational TCF for nanoconfined water which shows a slightly faster decay than the bulk. The distinction is prominent, especially for $R_{c}=1 \mathrm{~nm}$. However, $C_{1}(t)$ converges to the bulk response with increasing cavity size. (c) Total DMTCFs of SF inside spherical nanocavities show approximately four times faster relaxation. (d) First rank orientational TCF for nanoconfined SF. Unlike water, the confined particles show a slower decay, however, with comparable timescales.

of water, (ii) hydrogen bond excitation, and (iii) the large force constant of polarization free energy fluctuation [13]. Singleparticle rotation contributes at later stages $(\sim 10 \%-20 \%)$ $[43,44]$. We calculate collective orientational correlations (total dipole moment TCF, DMTCF) $\left[C_{M}(t)\right]$ and single-particle rotational correlations $\left[C_{1}(t)\right]$ for confined liquids [Eq. (9)].

$$
C_{M}(t)=\langle\boldsymbol{M}(0) \cdot \boldsymbol{M}(t)\rangle ; \quad C_{1}(t)=\frac{1}{N} \sum_{i=1}^{N}\left\langle\cos \left(\widehat{\boldsymbol{\eta}}_{0}^{i} \cdot \widehat{\boldsymbol{\eta}}_{t}^{i}\right)\right\rangle .
$$

Here, $\hat{\eta}_{t}^{i}$ is the unit vector along the molecular dipole of the $i$ th molecule at time $t$. We observe 20 times faster relaxation of DMTCF for nanoconfined water than the bulk [Fig. 3(a)]. This observation remains independent of the chosen surface-liquid interaction and cavity size. Bulk $C_{M}(t)$ exhibits exponential decay with an approximately $10 \mathrm{ps}$ component. Experimentally it is $\sim 8.3 \mathrm{ps}[45]$. On the other hand, $C_{M}(t)$ of cavity water molecules exhibit biexponential decay with timescales $\sim 30 \mathrm{fs}(40 \%)$ and $\sim 700 \mathrm{fs}(60 \%)$. We also observe bimodal relaxation in $C_{1}(t)$ : (i) $\sim 200-600 \mathrm{fs}$ regime $(10 \%-20 \%)$ and (ii) $\sim 3-4$ ps regime $(80 \%-90 \%)$. However, these timescales are comparable to the bulk relaxation [Fig. 3(b)]. Figures 3(c) and 3(d) show $C_{M}(t)$ and $C_{1}(t)$ for confined SF: $C_{M}(t)$ relaxes approximately four times faster than the bulk; but $C_{1}(t)$ is slower than the bulk, although with comparable timescales. Blaak and Hansen had earlier reported faster relaxation of $C_{M}(t)$ for Stockmayer fluid [19] (see SM S6 [35] for timescales).

The reason for such anomalous ultrafast decay can be traced to two factors: (i) inward propagation of surfaceinduced orientational correlations and (ii) the interplay between self-correlations and cross correlations of different regions inside the cavity. We divide the system into two, four, and eight equal regions to obtain region-specific dipole moments. The time trajectories of local dipoles reveal the 

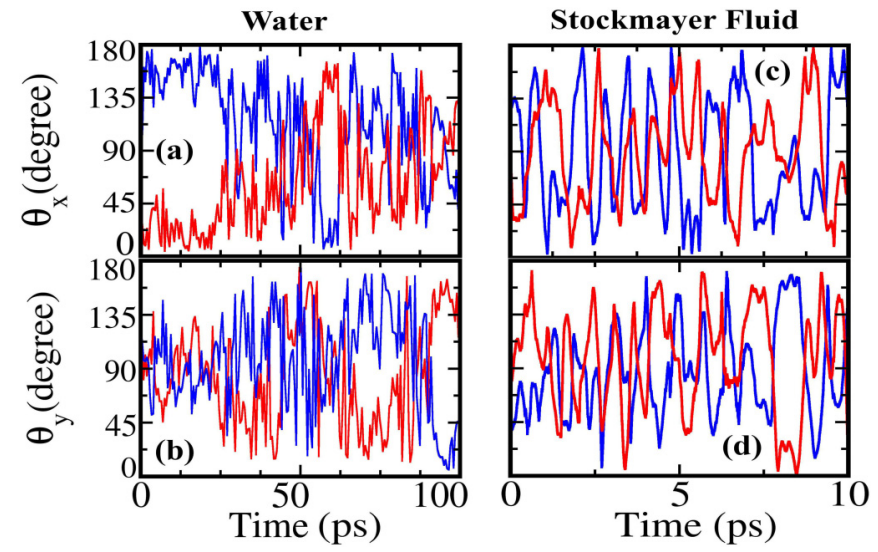

FIG. 4. Time evolution of angles created by the total dipole moment vectors of two hemispheres with (a) $X$ axis and (b) $Y$ axis for an $R_{c}=4 \mathrm{~nm}$ aqueous system with atomistic LJ-12,6 walls. (c) and (d) show similar plots for an $R_{c}=4 \mathrm{~nm}$ SF system with LJ-9,3 walls. In SF, the fluctuations are short lived. These plots show strong anticorrelated dipole flips that result in enormous cancellations. Blue and red represent northern and southern hemispheres, respectively.

correlation length in the system. In Fig. 4, we plot the time trajectories of angles made by the total dipole moment vectors of two hemispheres with Cartesian axes for $R_{c}=4 \mathrm{~nm}$. For water, we observe anticorrelated flips for two of the direction cosines associated with a Pearson's correlation coefficient $\left(\rho_{i j}\right) \sim-0.85$, where, $i$ and $j$ indicate two different regions (here, two hemispheres). However, along the third axis correlation is weaker $\left(\rho_{i j} \sim 0.18\right)$. We observe similar trends for other systems as well (SM S7) [35]. However, for $\mathrm{SF}$, such anticorrelations are rather weak $\left(\rho_{i j} \sim-0.3-0.4\right)$ and short lived. Additionally, we observe anticorrelations in direction cosines for concentric spheres of smaller radii inside a nanocavity (SM S7) [35].

Next, we dissect the total DMTCF in terms of subensembles. This reveals the timescales of anticorrelations. If we divide the sphere into " $m$ " equal subensembles, there arise $m$ number of self-terms $\left(\left\langle M_{i}(0) M_{i}(t)\right\rangle\right)$ and ${ }^{m} P_{2}=\frac{m(m-1)}{2}$ number of cross terms $\left\langle M_{i}(0) M_{j}(t)\right\rangle$ [Eq. (10)].

$$
\begin{aligned}
M(t) & =\sum_{i=1}^{m} M_{i}(t) ; \quad \text { and } \\
\langle M(0) M(t)\rangle & =\sum_{i=1}^{m}\left\langle M_{i}(0) M_{i}(t)\right\rangle+\sum_{i, j=1}^{m}\left\langle M_{i}(0) M_{j}(t)\right\rangle .
\end{aligned}
$$

Figure 5(a) (for $R_{c}=4 \mathrm{~nm}$ and $m=8$ ) demonstrates the presence of anticorrelation among the dipole moments of eight grids. For $m=2$ and 4 we observe similar behavior. Although the amplitudes of self-terms are approximately four to ten times larger than that of the cross terms, the total negative contribution arising from the anticorrelated terms makes the resultant decay ultrafast [red dashed curves in Fig. 5(a)]. Furthermore, the power spectrum of DMTCF for nanoconfined water exhibits bimodal $1 / f$ noise [Fig. 5(b)]. We attribute the deviation from bulk exponent to the surface effect and heterogeneous dynamics inside the nanocavities.
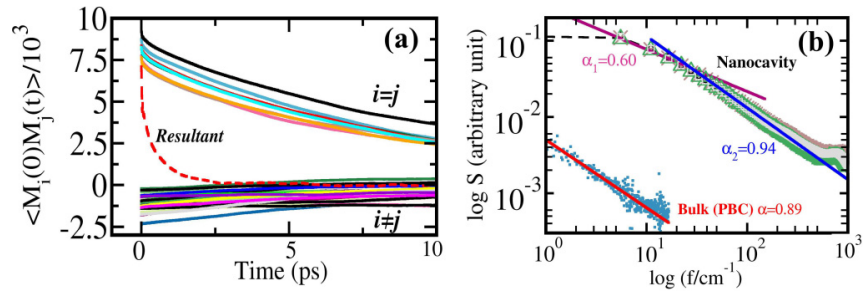

FIG. 5. (a) Self- and cross-DMTCF among eight grids inside an aqueous nanocavity of $R_{c}=4 \mathrm{~nm}$. The amplitudes of self-terms are approximately four to ten times higher than that of the cross terms. However, there are 8 self- and 56 cross terms that construct $\langle M(0) M(t)\rangle$. As a result, the negative contributions from anticorrelated cross terms predominate at longer times. This makes the net relaxation (red dashes) drastically fast. (b) Bimodal $1 / f$ character of the power spectrum of dipole moment fluctuation under confinement.

In order to rationalize the results shown in Figs. 4 and 5, we compute the Kirkwood $g$ factor $\left(g_{K}\right)$ [Eq. (11a)] for nanocavities and bulk. $g_{K}$ reveals information on the microscopic ordering of molecular dipoles. $g_{K}=1$ for completely random orientations.

$$
\begin{aligned}
& g_{K}=\frac{\left\langle M^{2}\right\rangle}{N \mu^{2}} \\
& \tau_{M}=\frac{g_{K}}{g_{K}^{D}(0)} \tau_{S} .
\end{aligned}
$$

We provide the numerical values of $g_{K}$ in SM S8 [35]. $g_{K}$ for confined water varies between 0.15 and 0.21 , whereas in the bulk (PBC) $g_{K}$ is $\sim 3.6$ at $300 \mathrm{~K}$. In an earlier study on water clusters, Saito and Ohmine reported similar low $g_{K}$ [17].

The timescale of DMTCF $\left(\tau_{M}\right)$ is related to $g_{K}$ and singleparticle rotational correlation timescale $\left(\tau_{S}\right)$ by Eq. $(11 \mathrm{~b})$, where $g_{K}^{D}(0)$ is the dynamic Kirkwood $g$ factor [46]. We find a substantial reduction of $g_{K}$ under confinement compared to the bulk, irrespective of the size of the cavities. For water it becomes almost 20 times smaller than the bulk. However, for SF, it becomes approximately four times smaller than the bulk. This indicates that the collective alignment of microscopic dipoles is destructive inside spherical nanocavities resulting in enormous cancellations among correlations. This is due to inward propagation of surface-induced correlations that interfere destructively. On the other hand, in periodic bulk systems, the microscopic dipoles align constructively. We obtain $g_{K}^{D}(0)$ in between 1.4 and 1.8 inside aqueous nanocavities and 1.5 for bulk SPC/E water. The deviation in $g_{K}^{D}(0)$ from the bulk is not significant. Hence, from Eq. (11b), $\tau_{M}$ becomes approximately proportional to $g_{K}$. This explains the faster collective relaxation inside the cavity. This is related to the observed blueshift and a faster decay of the perpendicular dielectric absorption spectrum by Gekle and Netz [40].

We perform layerwise analyses (thickness $\sim 5 \AA$ ) to study the differences in relative surface orientations as we approach the center. We obtain the distributions of angles formed between $\mathrm{O}-\mathrm{H}$ bonds of water and the surface normal. We observe a distinct peak around $\sim 90^{\circ}$ for the outermost water layer (SM S9) [35]. This demonstrates the existence of certain preferred orientations near the surface. Similar observations have been made by Ruiz-Barragan et al. from ab initio simulations of 
water inside graphene slit pores [47]. The water molecules follow the principle of minimal frustration often used to describe protein folding and spin-glass transitions $[28,29]$. In this case, it occurs through the maximization of hydrogen bonds in order to minimize the free energy. Banerjee et al. reported similar observations for a two-dimensional Mercedes-Benz model confined between two hydrophobic plates [48]. However, in SF we cannot make such distinctions between surface layers and interiors (SM S9) [35]. We find that aqueous solvation dynamics and water O-O-O angle distribution under confinement converge to the respective bulk pattern for smaller nanocavities (SM S10 and S11) [35].

\section{SUMMARY AND CONCLUSIONS}

The dielectric constant of a liquid is a collective property, determined by the long wavelength orientational correlations in the system [42,49]. Because of the long-ranged orientational correlations in dipolar liquids, the dielectric constant is rather strongly dependent on size and shape. Use of the PBC in simulations thus introduces an approximation which needs to be tackled carefully. The approach via the ClausiusMossotti equation is exact but one has to deal with a slow convergence. For water, this convergence is particularly slow because of the extensive HBN. As detailed in this Rapid Communication, the problem becomes more acute in the nanoworld.

The present study varies the size of the nanocavity and water-surface interactions giving rise to substantial alterations in the structure and dynamics of dipolar liquids. We find a substantial reduction in $\varepsilon$ of nanoconfined water at small cavity sizes $(\sim 2-3 \mathrm{~nm}$ radius $)$. The convergence toward the bulk value is surprisingly slow. However, $\varepsilon$ of Stockmayer liquid shows a relatively weaker dependence on the size of the nanocavity. We find that the Clausius-Mossotti equation is rather sensitive to the volume of a system. In the nanoscopic world volume is defined by intermolecular interactions, unlike the macroscopic description of volume, that is prescribed from outside. When the enclosing surface is modeled as soft spheres, effective volume calculation is subject to errors. We show that a small error in $V_{\text {eff }}$ leads to substantial changes in $\varepsilon$ for nanoconfined water, unlike SF. We explain the anomalous ultrafast relaxation in terms of substantially low values of Kirkwood $g$ factor and anticorrelated local dipole moments of different subensembles inside the cavity. The nature of the surface-liquid interaction alters the values of $\varepsilon$ but does not affect the general trends. We have confirmed this claim by using four other surface-water interactions (SM S12) [35]. Additionally, in SM S13, we present an Ising model based analysis that reproduces the quenched dipole moment fluctuations under a constrained environment [35].

\section{ACKNOWLEDGMENTS}

We thank Professor R. N. Zare for early collaboration. We also thank Professor Dominik Marx for pointing out the works of Netz and Hansen. B.B. acknowledges support from Sir J. C. Bose Fellowship and also acknowledges DST for partial support. S.M. acknowledges UGC and S.A. acknowledges IISc for scholarship.
[1] B. Bagchi, Water in Biological and Chemical Processes: From Structure and Dynamics to Function (Cambridge University Press, Cambridge, UK, 2013).

[2] K. Bhattacharyya and B. Bagchi, J. Phys. Chem. A 104, 10603 (2000).

[3] P. Jungwirth, J. Phys. Chem. Lett. 6, 2449 (2015).

[4] D. Muñoz-Santiburcio and D. Marx, Phys. Rev. Lett. 119, 056002 (2017).

[5] S. Mondal, S. Acharya, R. Biswas, B. Bagchi, and R. N. Zare, J. Chem. Phys. 148, 244704 (2018).

[6] J. K. Lee, S. Banerjee, H. G. Nam, and R. N. Zare, Quart. Rev. Biophys. 48, 437 (2015).

[7] I. Nam, J. K. Lee, H. G. Nam, and R. N. Zare, Proc. Natl. Acad. Sci. USA 114, 12396 (2017).

[8] R. Biswas and B. Bagchi, J. Chem. Phys. 133, 084509 (2010).

[9] S. Mondal, S. Mukherjee, and B. Bagchi, J. Phys. Chem. Lett. 8, 4878 (2017).

[10] S. Park, D. E. Moilanen, and M. D. Fayer, J. Phys. Chem. B 112, 5279 (2008).

[11] R. Biswas, N. Rohman, T. Pradhan, and R. Buchner, J. Phys. Chem. B 112, 9379 (2008).

[12] I. R. Piletic, D. E. Moilanen, D. Spry, N. E. Levinger, and M. Fayer, J. Phys. Chem. A 110, 4985 (2006).

[13] B. Bagchi, Molecular Relaxation in Liquids (Oxford University Press, New York, 2012).

[14] B. Bagchi, D. W. Oxtoby, and G. R. Fleming, Chem. Phys. 86, 257 (1984).
[15] B. Bagchi, Annu. Rev. Phys. Chem. 40, 115 (1989).

[16] L. Fumagalli, A. Esfandiar, R. Fabregas, S. Hu, P. Ares, A. Janardanan, Q. Yang, B. Radha, T. Taniguchi, K. Watanabe, G. Gomila, K. S. Novoselov, and A. K. Geim, Science 360, 1339 (2018).

[17] S. Saito and I. Ohmine, J. Chem. Phys. 101, 6063 (1994).

[18] S. Senapati and A. Chandra, J. Phys. Chem. B 105, 5106 (2001).

[19] R. Blaak and J.-P. Hansen, J. Chem. Phys. 124, 144714 (2006).

[20] A. Schlaich, E. W. Knapp, and R. R. Netz, Phys. Rev. Lett. 117, 048001 (2016).

[21] P. Debye, Polar Molecules (Chemical Catalog, New York, 1929).

[22] J. G. Kirkwood, J. Chem. Phys. 7, 911 (1939).

[23] C. Schaaf and S. Gekle, J. Chem. Phys. 145, 084901 (2016).

[24] V. Ballenegger and J.-P. Hansen, J. Chem. Phys. 122, 114711 (2005).

[25] C. Alba-Simionesco, B. Coasne, G. Dosseh, G. Dudziak, K. Gubbins, R. Radhakrishnan, and M. Sliwinska-Bartkowiak, J. Phys.: Condens. Matter 18, R15 (2006).

[26] G. Ping, J. Yuan, M. Vallieres, H. Dong, Z. Sun, Y. Wei, F. Li, and S. Lin, J. Chem. Phys. 118, 8042 (2003).

[27] J. C. Rasaiah, S. Garde, and G. Hummer, Annu. Rev. Phys. Chem. 59, 713 (2008).

[28] J. D. Bryngelson and P. G. Wolynes, Proc. Natl. Acad. Sci. USA 84, 7524 (1987). 
[29] J. N. Onuchic and P. G. Wolynes, Curr. Opin. Struct. Biol 14, 70 (2004).

[30] H. Fröhlich, Theory of Dielectrics (Clarendon, Oxford, 1949).

[31] C. J. F. Böttcher, O. C. van Belle, P. Bordewijk, and A. Rip, Theory of Electric Polarization (Elsevier Science, Ltd., 1978), Vol. 2.

[32] R. Kubo, J. Phys. Soc. Jpn. 12, 570 (1957).

[33] C. Schaaf and S. Gekle, Phys. Rev. E 92, 032718 (2015).

[34] D. J. Berendsen, Molecular dynamics and Monte Carlo calculations on water, CECAM Report (1972), p. 29.

[35] See Supplemental Material at http://link.aps.org/supplemental/ 10.1103/PhysRevResearch.1.033145 for Berendsen's derivation, effective volume calculation, simulation details, convergence test, sensitivity to density, relaxation timescales, Kirkwood $g$ factor, Pearson correlation coefficients, orientation of water molecules, tetrahedral angle distribution, solvation dynamics, effect of different surface-liquid potential, and an Ising model based explanation of the scaling of $\varepsilon$ with size.
[36] B. Felderhof, Phys. A (Amsterdam, Neth.) 101, 275 (1980).

[37] A. Chandra and B. Bagchi, J. Chem. Phys. 90, 1832 (1989).

[38] T. L. Hill, Thermodynamics of Small Systems (Courier Corporation, 1994).

[39] L. Wang and J. Hermans, Mol. Simul. 17, 67 (1996).

[40] S. Gekle and R. R. Netz, J. Chem. Phys. 137, 104704 (2012).

[41] S. Mondal and B. Bagchi, J. Phys. Chem. Lett. 10, 6287 (2019).

[42] A. Chandra and B. Bagchi, J. Chem. Phys. 91, 3056 (1989).

[43] B. Bagchi and B. Jana, Chem. Soc. Rev. 39, 1936 (2010).

[44] S. Mondal, S. Mukherjee, and B. Bagchi, J. Chem. Phys. 147, 154901 (2017).

[45] R. Buchner, J. Barthel, and J. Stauber, Chem. Phys. Lett. 306, 57 (1999).

[46] P. Madden and D. Kivelson, Adv. Chem. Phys. 56, 467 (1984).

[47] S. Ruiz-Barragan, D. Muñoz-Santiburcio, and D. Marx, J. Phys. Chem. Lett. 10, 329 (2019).

[48] S. Banerjee, R. S. Singh, and B. Bagchi, J. Chem. Phys. 142, 134505 (2015).

[49] B. Bagchi and A. Chandra, J. Chem. Phys. 90, 7338 (1989). 